Check for updates

Cite this: RSC Adv., 2021, 11, 39311

\title{
Airflow-assisted dielectrophoresis to reduce the resistance mismatch in carbon nanotube-based temperature sensors $\uparrow$
}

\author{
Abdullah Abdulhameed, (D) *ab Izhal Abdul Halin, ${ }^{\mathrm{b}}$ Mohd Nazim Mohtar (D) bc \\ and Mohd Nizar Hamidon ${ }^{\text {bc }}$
}

\begin{abstract}
The dielectrophoresis (DEP) method is used to fabricate sensor devices by assembling and aligning carbon nanotubes (CNTs) across electrode structures. The challenges of the method increase as the gap width between the electrodes increases. In this work, a novel DEP setup is proposed to reduce the resistance mismatch in manufacturing carbon nanotube-based sensors. The proposed setup utilizes hot airflow and thermal annealing to fabricate long-aligned multi-walled carbon nanotube (MWCNT) bridges across transparent electrodes with a gap width up to $75 \mu \mathrm{m}$. The best alignment results were obtained at airflow velocities between $1.5 \mathrm{~m} \mathrm{~s}^{-1}$ and $2.5 \mathrm{~m} \mathrm{~s}^{-1}$. The minimum variation in the resistance of the aligned bridges was $1.81 \%$ observed at a MWCNT concentration of $0.005 \mathrm{wt} \%$ and deposition time of $10 \mathrm{~min}$. Long MWCNT bridges have many contact points that link MWCNTs to each other, making the contact resistance a robust indicator of the variation in the ambient temperature. The characteristics of the MWCNT bridges as a temperature sensor, including the response, sensitivity, and recovery, were investigated.
\end{abstract}

Received 10th November 2021 Accepted 30th November 2021

DOI: $10.1039 / \mathrm{d} 1 \mathrm{ra0} 8250 \mathrm{~g}$

rsc.li/rsc-advances

CNTs are also used to sense environmental data such as

\section{Introduction}

The field of sensor research is very broad. However, in recent years, the focus has been on producing low-cost, flexible, and transparent sensors with the ability to integrate them into future healthcare and portable electronics. ${ }^{1,2}$ Low-cost production of sensors includes the usage of cheap methods such as printing sensors on papers using carbon ink or utilizing electric fields to deposit carbon nanostructures on microelectrodes at room temperature. ${ }^{3,4}$ Carbon nanostructures such as graphene nanoribbon (GNR) and carbon nanotubes (CNTs) are widely used as sensing materials due to their physical dimensions and chemical stability. ${ }^{5,6}$

The remarkable optical, mechanical, and electrical properties of CNTs allowed them to be used in various applications. ${ }^{7,8}$ For example, in gas sensor applications, CNTs can detect gas molecules at low concentrations, such as the detection of ammonia and hydrogen., ${ }^{9,10}$ In biosensor applications, CNTs have the ability to sense heated bacteria and COVID19 virus. ${ }^{11,12}$

${ }^{a}$ Department of Electronic Engineering, Faculty of Engineering, Hadhramout University, Mukalla, Yemen.E-mail: gs50667@student.upm.edu.my

${ }^{b}$ Department of Electrical and Electronic Engineering, Faculty of Engineering, Universiti Putra Malaysia, Serdang, 43400, Malaysia

'Institute of Advanced Technology (ITMA), Universiti Putra Malaysia, Serdang, 43400, Malaysia

$\dagger$ Electronic supplementary information (ESI) available. See DOI: 10.1039/d1ra08250g temperature and humidity. ${ }^{13}$ However, ambient temperature is the most frequently measured variable in daily life, monitor systems, and industrial applications. ${ }^{14}$

The high stability and low power consumption made CNTs suitable to work as temperature sensors. ${ }^{15}$ Moreover, CNTs can operate in a wide temperature range without being physically affected. Several sensing mechanisms were reported to theoretically explain the change in the CNT resistance following the change in the ambient temperature. ${ }^{16}$ Most of the studies reported negative temperature coefficients (TCs), where the sensor resistance decreases as the temperature increases. ${ }^{17,18}$ On the other hand, positive TCs were also reported, where the sensor resistance increases with the temperature increase. ${ }^{19}$ Kuo et al. related the change in the resistance to the nature of the CNTs. ${ }^{19}$ They concluded that the resistance is directly proportional to the temperature in the case of CNTs with metallic nature and inversely in the case of CNTs with semiconducting nature. The same study showed that the gap width between the sensor electrodes significantly affects the sensor response.

The sensing material in temperature sensors is either solo CNTs or a composite of CNTs and other materials. In the first case, limited studies addressed sensors that use only CNTs (either single-walled carbon nanotubes (SWCNTs) or multiwalled carbon nanotubes (MWCNTs)) to detect temperature variation. ${ }^{20,21}$ In the second case, the sensing material was manufactured by mixing CNTs with other materials such as 
epoxy resin, ${ }^{22}$ poly(vinylidene fluoride) (PVDF), ${ }^{23}$ graphene oxide, ${ }^{24}$ silicon, ${ }^{25}$ and PEDOT:PSS. ${ }^{26}$ In both cases, CNTs are preferred to be aligned in order to achieve their best performance.

The inability to produce aligned CNTs across the sensor electrodes is the major limitation in manufacturing CNT-based sensors. Using random CNT networks produces devices with anisotropic electrical properties with considerable variation in their resistance. ${ }^{27}$ Fabrication of temperature sensors using methods such as screen printing, ${ }^{28}$ ink printing, ${ }^{29}$ additive printing, ${ }^{30}$ doctor blade, ${ }^{31}$ spinning process, ${ }^{32}$ chemical vapor deposition (CVD), ${ }^{33}$ and vacuum filtration ${ }^{34}$ consume large amounts of CNTs, making them costly. Moreover, the repeatability of the manufacturing process produces sensors with different resistance due to the low alignment quality, which causes a mismatch in the sensor performance. ${ }^{35}$ Consequently, this would lead to necessitating individual calibration of each sensor. ${ }^{36}$

Dielectrophoresis (DEP) is one of the sensors' fabrication methods that is used to overcome the isotropic properties of CNTs by assembling them onto the device in an aligned form. ${ }^{37}$ DEP does not require high specific setups compared with the mentioned methods. However, DEP is restricted to the device geometry where there are difficulties in maintaining the alignment quality across gaps with large-scale dimensions due to the poor controllability of the method..$^{38}$ Improving the DEP setup while maintaining its simplicity is expected to preserve the alignment quality and thus reduce the resistance variation that might occur due to the distortion in the aligned CNTs.

In this article, a novel DEP setup is proposed to fabricate CNT-based temperature sensors. The setup utilizes airflow with a specific velocity and temperature to assist the alignment of long MWCNT bridges across transparent electrodes. The shear force generated by the airflow is expected to produce a torque on the tubes to maintain their alignment quality. In addition to the alignment, airflow simultaneously spreads the solution droplet and helps in the drying process. The new setup is expected to minimize the mismatch in the resistance of the fabricated temperature sensors. The article also investigates the characteristics and performance of the temperature sensors in terms of sensitivity, response, and recovery.

\section{Materials and methods}

\subsection{Electrode fabrication}

The lithography method was used to fabricate indium tin oxide (ITO) electrodes on a glass substrate. First, the ITO layer was spin-coated with a positive photoresist and then dried on a hotplate at a temperature of $90{ }^{\circ} \mathrm{C}$ for 2 minutes. Next, the dried photoresist was covered with a photomask and exposed to UV light for 7 seconds. The photomask was a design of interdigitated electrodes (IDE) with different gap widths. The substrate was then soaked in a developer to dissolve the photoresist pattern that was exposed to the UV light then dried in an oven at $120^{\circ} \mathrm{C}$ for 2 hours. Finally, a wet etching procedure was conducted to etch the ITO using a mixture of hydrochloric acid and nitric acid $(4: 1)$. The fabricated devices were cleaned using IPA, acetone, and DIW. The fabrication steps are illustrated in Fig. S1. $\uparrow$ Pre-fabricated electrodes with gap widths of $50 \mu \mathrm{m}$ and $75 \mu \mathrm{m}$ (Ossila Ltd, UK) were also used in this work.

\subsection{Suspensions preparation}

Table 1 lists the samples that were used in the fabrication to form the sensing layer. The first sample was a pristine MWCNTs obtained from Shenzhen Nanotechnologies (Shenzhen, China) with an average length of $10 \mu \mathrm{m}$ and an average diameter of $30 \mathrm{~nm}$. The MWCNTs were functionalized using mild acid treatment to increase their solubility and dispersity in solvents. The mild acid treatment procedure used to functionalize this sample was explained elsewhere. ${ }^{39}$ The second and third samples were commercial MWCNTs (Cheaptubes, US) functionalized with $\mathrm{COOH}$ and $\mathrm{OH}$, respectively. The fourth sample was a mixture of MWCNTs and conductive amorphous carbon (CAC) functionalized with nitric acid, as described elsewhere. ${ }^{\mathbf{4 0}}$ One $\mathrm{mg}$ of each sample was added to $20 \mathrm{~mL}$ of dimethylformamide (DMF) at different concentrations. The solutions were sonicated for 30 minutes in a cold bath to form MWCNT suspensions. The MWCNT suspensions were stored for two weeks at room temperature in order to let large bundles settle down. Then, the supernatant was collected and further sonicated for 5 minutes before using it in the dielectrophoretic assembly. The preparation procedures of the suspensions are illustrated in Fig. S2.†

\subsection{Dielectrophoresis setup}

Fig. 1 shows the schematic of the airflow-assisted dielectrophoresis (AA-DEP). The glass substrate was placed on doublesided tape with an angle of $20^{\circ}$ at different distances from an air gun. The airflow velocity and temperature were recorded at these distances (see Fig. S3†). The dielectrophoretic assembly of MWCNTs was conducted by pipetting $20 \mu \mathrm{L}$ of the MWCNT suspensions on the IDE electrodes. After making sure the droplet covers all IDE fingers, an AC signal of $20 \mathrm{~V}_{\mathrm{pp}}$ and 2.5 $\mathrm{MHz}$ was applied across the electrodes for 10 minutes. Then, a dry absorbance paper was used to absorb and remove the droplet without switching off the AC generator. Hot airflow with a velocity of $2 \mathrm{~m} \mathrm{~s}^{-1}$ and a temperature of $50{ }^{\circ} \mathrm{C}$ was used to push away the remaining drop and dry the assembled MWCNT bridges. The hot airflow is expected to maintain the alignment quality while speeding up the drying process by evaporating the remaining medium. The fabricated sensors were thermally annealed on a hotplate for 1 hour at a temperature of $250^{\circ} \mathrm{C}$ to

Table 1 Samples that were used as sensing layers in the fabricated sensors

\begin{tabular}{lll}
\hline Sensor code & Sensing layers (samples) & Functional group \\
\hline SC1 & MWCNTs & OH \\
SC2 & MWCNTs & COOH \\
SC3 & MWCNTs & OH $+\mathrm{COOH}$ \\
SC4 & MWCNTs + CAC & OH $+\mathrm{COOH}$
\end{tabular}




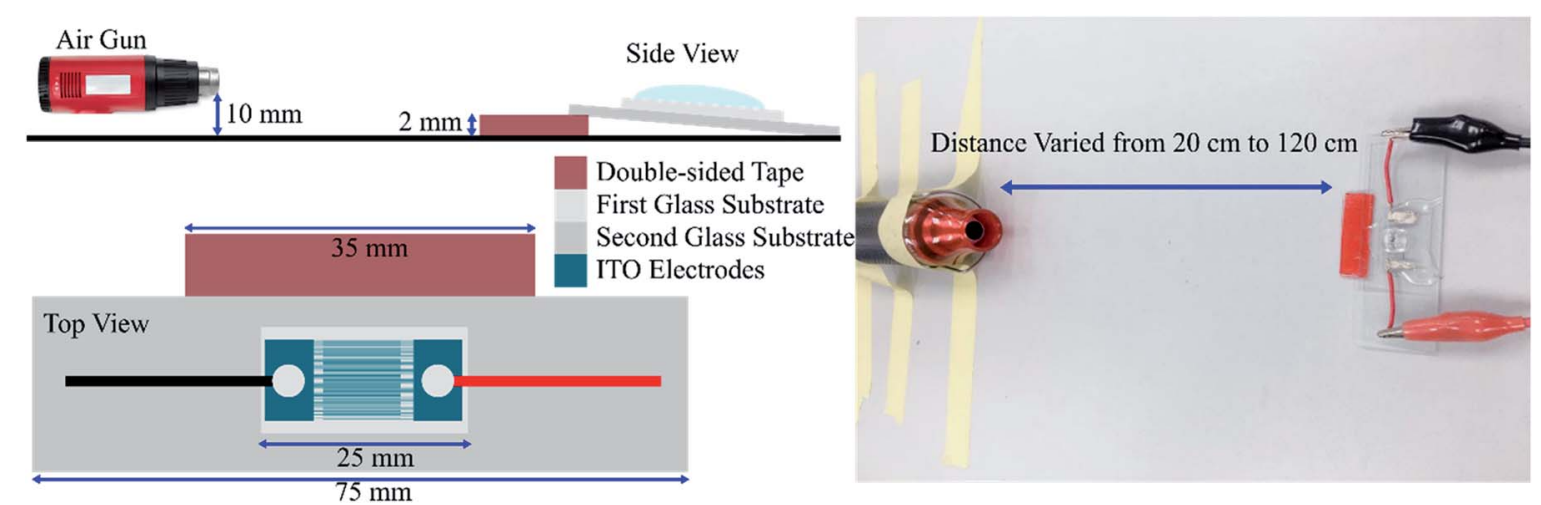

Fig. 1 The setup of the AA-DEP. The distance between the air gun and the substrate was varied to control the velocity and temperature of the airflow. Alligator probes were connected to ITO electrodes to supply the system with an AC signal. The signal generator was connected to a PC to control the parameters of the signal.

stabilize the contact resistance between the tubes. The initial resistance of the sensors was recorded at room temperature one day after the annealing.

\subsection{Sensor characterization}

Two sensing setups were used to test the sensor response to the temperature changes. In the first setup, the sensors were placed on a digital hotplate and connected to a multimeter. The multimeter was connected to a PC to log the resistance data and characterize the sensors in real-time. Environmental effects such as humidity and wind speed have a significant effect on the sensitivity of the carbon-based sensors. ${ }^{41}$ Thus, the sensors were covered with aluminum foil to ensure minimum ambient environmental effects and obtain a reliable response of the long MWCNT bridges. The hotplate temperature varied from $30^{\circ} \mathrm{C}$ to $70{ }^{\circ} \mathrm{C}$, and the change (increase/decrease) in the sensor resistance was recorded. In the second setup, a hot airflow gun was pointed to the back of the glass substrate. The air temperature was measured, and the sensor's resistance was recorded as a function of time. The air gun provides fast heating and allows the sensors to recover to room temperature faster than the hotplate. Fig. S4 in the ESI $\uparrow$ illustrates the testing process of the sensors.

\section{Results and discussion}

The main objective of this study is to improve the reproducibility in manufacturing CNT-based sensors using the proposed airflow-assisted DEP method. The reason for using different samples of CNTs is to strengthen the result and prove the usability of the proposed method. The characterization results of the used samples can be found in the ESI. $\dagger$ FTIR, UV-Vis FESEM, and HRTEM of the MWCNTs and CAC are shown in Fig. S5 and S6. $\dagger$ Further details can be found elsewhere. ${ }^{39,40}$

\subsection{Dielectrophoretic assembly}

One reason that causes the resistance mismatch is the distortion in the aligned bridges due to the dying process. The degree of distortion in the aligned bridges can be identified by measuring their resistance because each bridge represents a parallel resistance. Fig. 2a presents the velocity of the airflow and the resulting sensor resistance. In the case of a gap width of $50 \mu \mathrm{m}$, the variation in resistance was small at airflow velocities between 1.5 and $2.5 \mathrm{~m} \mathrm{~s}^{-1}$. However, for a gap width of $75 \mu \mathrm{m}$, airflow velocities lower than $1.5 \mathrm{~m} \mathrm{~s}^{-1}$ were ineffective, while velocity higher than $2.5 \mathrm{~m} \mathrm{~s}^{-1}$ destroyed some of the MWCNT bridges, causing an increase in the sensor resistance. Fig. S7 in the ESI $\dagger$ shows the effect of the airflow velocity on aligned MWCNT bridges across a gap width of $75 \mu \mathrm{m}$.

The reproducibility of the AA-DEP method was studied by measuring the variation in the resistance of a batch of sensors (five sensors in each case) fabricated at identical conditions. The variation in the sensor resistance (mean value and standard deviation) was investigated at different fabrication conditions, such as different MWCNT concentrations, deposition times, and sensing materials. Fig. $2 \mathrm{~b}$ shows the variation in the sensors' resistance as a function of MWCNT concentration in the suspension. The variations in the resistance of the sensors were $4.89 \%, 6.06 \%$, and $1.81 \%$ at MWCNT concentrations of $0.002,0.003$, and $0.005 \mathrm{wt} \%$, respectively. The minimum resistance mismatch was observed at the highest concentration.

Fig. 2c shows the variation in the sensors' resistance as a function of deposition time. The variations in the resistance were $12.39 \%, 8.05 \%$, and $4.89 \%$ at a deposition time of 3,5 , and 10 minutes. The minimum resistance mismatch was observed at the longest deposition time. The AA-DEP's ability to reduce the resistance mismatch was also tested for sensors fabricated using MWCNTs with different functional groups. Fig. 2d shows that the variations in the resistance were $4.89 \%, 2.914 \%$, and $5.284 \%$ for MWCNTs functionalized with $\mathrm{COOH}+\mathrm{OH}, \mathrm{COOH}$, and $\mathrm{OH}$, respectively. In conclusion, the AA-DEP setup successfully reduced the resistance mismatch by avoiding the distortion and deformation that might occur during the drying process. The minimum resistance variation was observed at a concentration of $0.005 \%$, and the worst resistance mismatch was observed at a deposition time of 3 minutes (resistance variation in basic alignment setups could exceed $10 \%){ }^{42}$ 

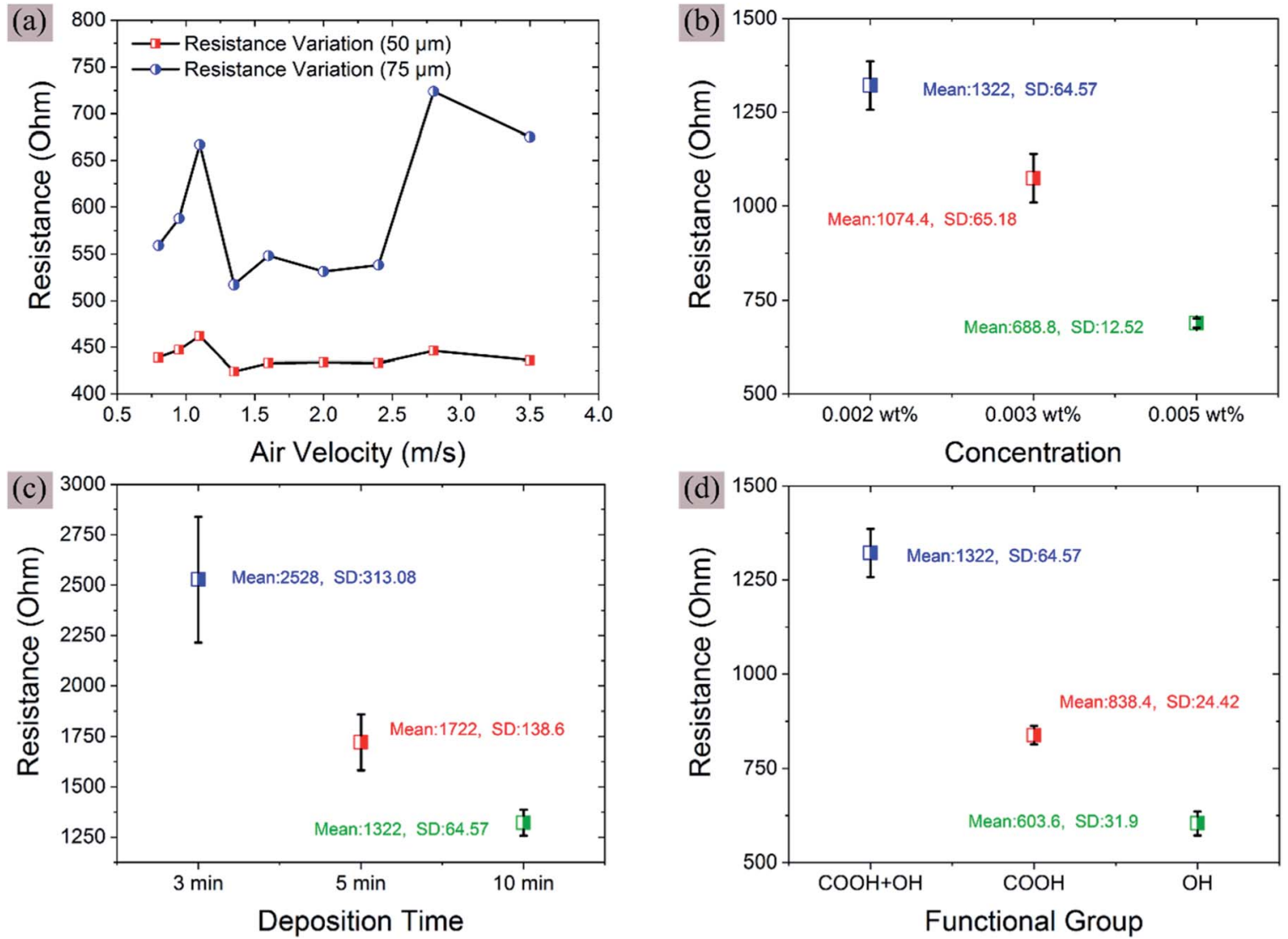

Fig. 2 Resistance variation in sensors fabricated using the AA-DEP setup. (a) Resistance of the aligned bridges versus airflow velocity at different gap widths (time: $10 \mathrm{~min}$, concentration: $0.005 \mathrm{wt} \%$, sample: SC1) (b) resistance variation at different MWCNT concentrations (time: 10 min, sample: SC3). (c) Resistance variation at different deposition times (concentration: $0.002 \mathrm{wt} \%$, sample: SC3). (d) Resistance variation using sensing material with different functional groups (time: $10 \mathrm{~min}$, concentration: $0.002 \mathrm{wt} \%$ ). (b-d) Electrode gap $50 \mu \mathrm{m}$, airflow velocity $2 \mathrm{~m} \mathrm{~s}{ }^{-1}$.

Fig. 3a shows an example of the fabricated transparent sensor using the AA-DEP setup. The thin dark layer across the ITO electrodes is the deposited MWCNTs. The darkness (density/thickness) of the layer and its resistance were controlled by controlling the fabrication parameters, especially the AC signal amplitude and duration (deposition time). Further details regarding how to control the density of the aligned MWCNTs can be found elsewhere. ${ }^{43}$ Fig. $3 \mathrm{~b}$ shows that

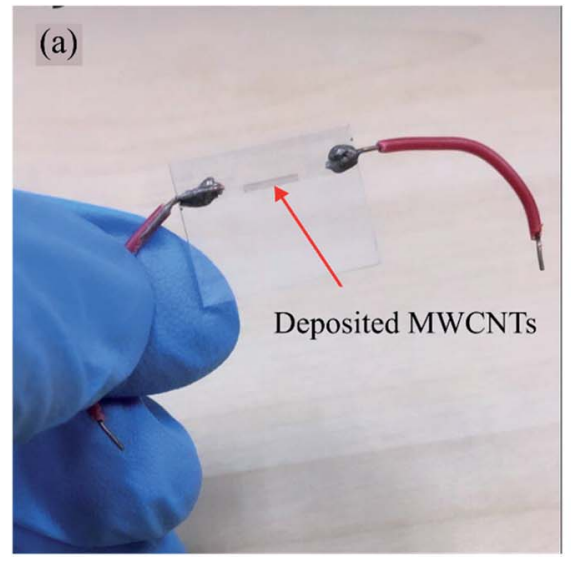

(b)

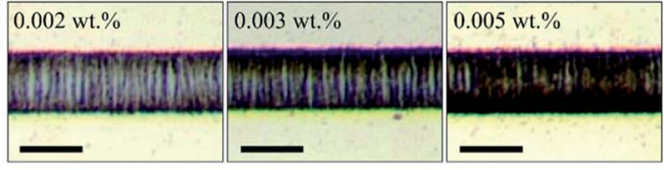

(c)
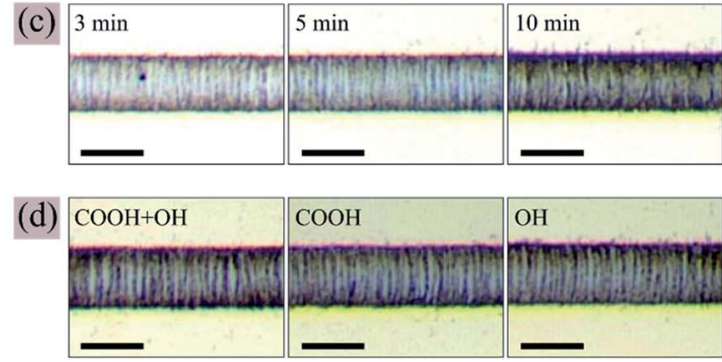

Fig. 3 Deposited MWCNTs across ITO electrodes. (a) The fabricated sensor. (b) Deposition of MWCNTs at different concentrations (time: 10 min, sample: SC3). (c) Deposition of MWCNTs at different deposition times (concentration: 0.002 wt\%, sample: SC3). (d) Deposition of MWCNTs functionalized with different functional groups (time: $10 \mathrm{~min}$, concentration: $0.002 \mathrm{wt} \%$ ) [scale bar: $50 \mu \mathrm{m}$, Optical microscope: Moticam, BA310E]. Large-scale and high-resolution images can be found in the ESI (Fig. S8 and S9†). 
the density of the deposited MWCNTs is directly proportional to the concentration of the MWCNTs in the medium. Thus, mediums with high concentration MWCNTs can be used to deposit a high-density MWCNT layer. At constant concentration, the density can also be controlled by the deposition time, as shown in Fig. 3c. The devices shown in Fig. 3d have the same density even though different types of functional groups were attached to the MWCNTs, which indicates that the functional groups on the MWCNT walls have less significant effects on the density compared with the deposition time and concentration. Although the density of the MWCNTs looks similar in Fig. 3d, the three devices have different resistance (refer to Fig. 2d).

\subsection{Sensor characterization}

The temperature coefficient of resistance (TCR) was used to describe the sensitivity and performance of the sensors. TCR relates the relative changes (increase/decrease) in the sensor resistance with the changes in the ambient temperature and can be calculated by eqn (1). ${ }^{44}$

$$
\alpha=\frac{R-R_{0}}{R_{0}\left(T-T_{0}\right)} 100 \%\left[{ }^{\circ} \mathrm{C}^{-1}\right]
$$

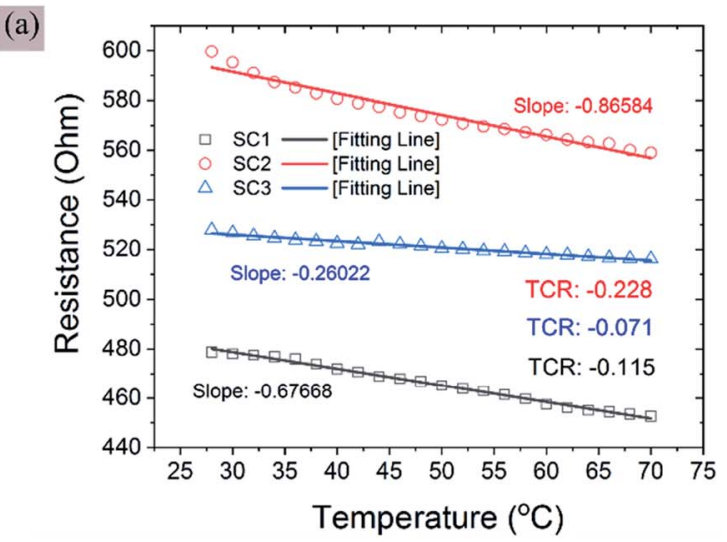

The TCR equation is equivalent to the slope of the fitting line of the experimental data over the initial sensor resistance. Thus, the sensor with a higher absolute TCR value indicates strong sensitivity. The experimental result showed that the sensitivity of the aligned MWCNT bridges depends on several factors, such as the density of the aligned bridges and the type of the functional group attached to the MWCNT walls.

Fig. 4a shows the response of MWCNT-based temperature sensors that differ from each other based on the functional group attached to their walls. The sensors that were fabricated using sample SC2 showed the strongest sensitivity with a TCR of $-0.228 \%{ }^{\circ} \mathrm{C}^{-1}$, while the worst sensitivity was observed in sensors fabricated using sample SC3 with TCR of $-0.07 \%{ }^{\circ} \mathrm{C}^{-1}$. The negative signal means the sensor resistance decreases as the temperature increases. Four reasons explain the difference in the sensitivity of the sensors: sample electrical conductivity, sample solubility in DMF, sample treatments, and the contact resistance (MWCNT-MWCNT/MWCNT-ITO). In terms of electrical conductivity, MWCNTs have different electrical conductivities based on the functional groups attached to their walls. ${ }^{45}$ For example, the sensor fabricated using sample SC1 has a TCR value higher than the sensor fabricated using sample SC3 $\left(|-0.115|>|0.07| \%{ }^{\circ} \mathrm{C}^{-1}\right)$ because the electrical conductivity of sample SC1 was higher than sample SC3. In terms of solubility,

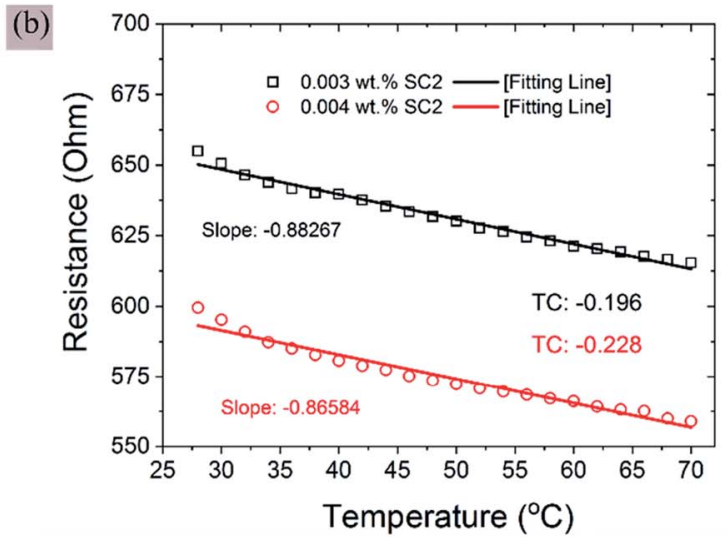

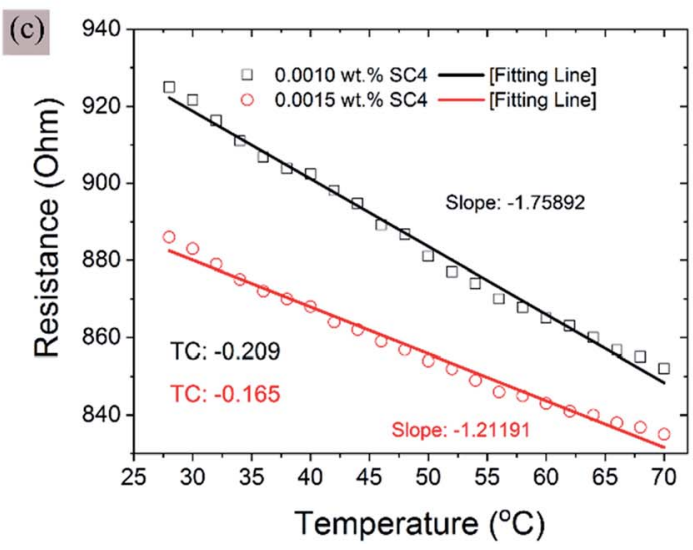

Fig. 4 Response of MWCNT-based temperature sensors. (a) Response of sensors that were fabricated using MWCNTs with different functional groups (fabrication parameters: $20 \mathrm{~V}, 10 \mathrm{~min}$ ). (b) Response of sensors that were fabricated using suspensions with different concentrations of MWCNTs (fabrication parameters: $20 \mathrm{~V}, 10 \mathrm{~min}, \mathrm{SC} 2$ ). (c) Response of sensors that were fabricated using a mixture of MWCNTs and amorphous carbon at two different concentrations (fabrication parameters: $20 \mathrm{~V}, 10 \mathrm{~min}$ ). 
MWCNTs functionalized with $\mathrm{COOH}$ showed better solubility in DMF, which results in better alignment thus strong sensitivity. MWCNTs that were treated or functionalized with more than one functional group have low quality due to the harsh treatment, which explains why sensor SC3 shows the worst sensitivity. Finally, the MWCNT-MWCNT and MWCNT-ITO contact resistance plays a critical role in the sensors' behavior. However, the contact resistance also depends on the quality of the MWCNTs and their dispersity in mediums. ${ }^{46}$

Fig. $4 \mathrm{~b}$ shows the response of three sensors fabricated under the same conditions using sample SC2 with different MWCNT concentrations. The range of the operating resistance of the sensors increased with the increase of the MWCNT concentration in the suspension. AA-DEP assembled more MWCNTs from the suspension with high MWCNT concentration, resulting in highdensity MWCNT bridges with low resistance. Although the slope of the linear fitting lines was convergent, the TCR of the sensors was different at different concentrations $\left(-0.12725 \%{ }^{\circ} \mathrm{C}^{-1}\right.$ at $0.003 \mathrm{wt} \%$ and $-0.19572 \%{ }^{\circ} \mathrm{C}^{-1}$ at $\left.0.004 \mathrm{wt} \%\right)$.

Fig. 4c shows the performance of sensors fabricated using a mixture of MWCNTs and CAC (SC4). The slope of the fitting lines was steeper than the fitting lines of the sensors that were fabricated using MWCNTs only (Fig. 4b). However, the TCR value was $-0.20984 \%{ }^{\circ} \mathrm{C}^{-1}$ at a concentration of $0.0015 \mathrm{wt} \%$ and $-0.16504 \%{ }^{\circ} \mathrm{C}^{-1}$ at a concentration of $0.0010 \mathrm{wt} \%$. Although the concentration of SC4 was much lower than the concentration of SC1-SC3, the TCR value was higher, which indicates that the spherical conductive carbon particles played a role in reducing the contact resistance between the tubes.

The sensors' behavior after annealing was entirely different in contrast to the behavior before annealing. The sensor resistance increased with the increase of the temperature. Fig. 5 shows the response of the sensor after exposing it to three cycles of hot airflow. The airflow temperature at the back of the glass substrate was $\sim 60{ }^{\circ} \mathrm{C}$. In sensor SC2, the resistance dropped from $543.8 \Omega$ (the initial resistance after the alignment and before annealing) to $529.5 \Omega$ in the first 200 seconds. However, the sensor resistance recovered to only $530.4 \Omega$ after 200 seconds from switching off the hot air gun. The sensor minimum and maximum resistance continued decreasing in the following cycles (Fig. 5a). The behavior of the sensor was stable after the annealing process, where the variation in the minimum and maximum resistance was very small. The sensor resistance increased from $443.6 \Omega$ to $447.41 \Omega$. The response cycles of SC3 after annealing were similar to those of SC2, with a significant decrease in the minimum and maximum
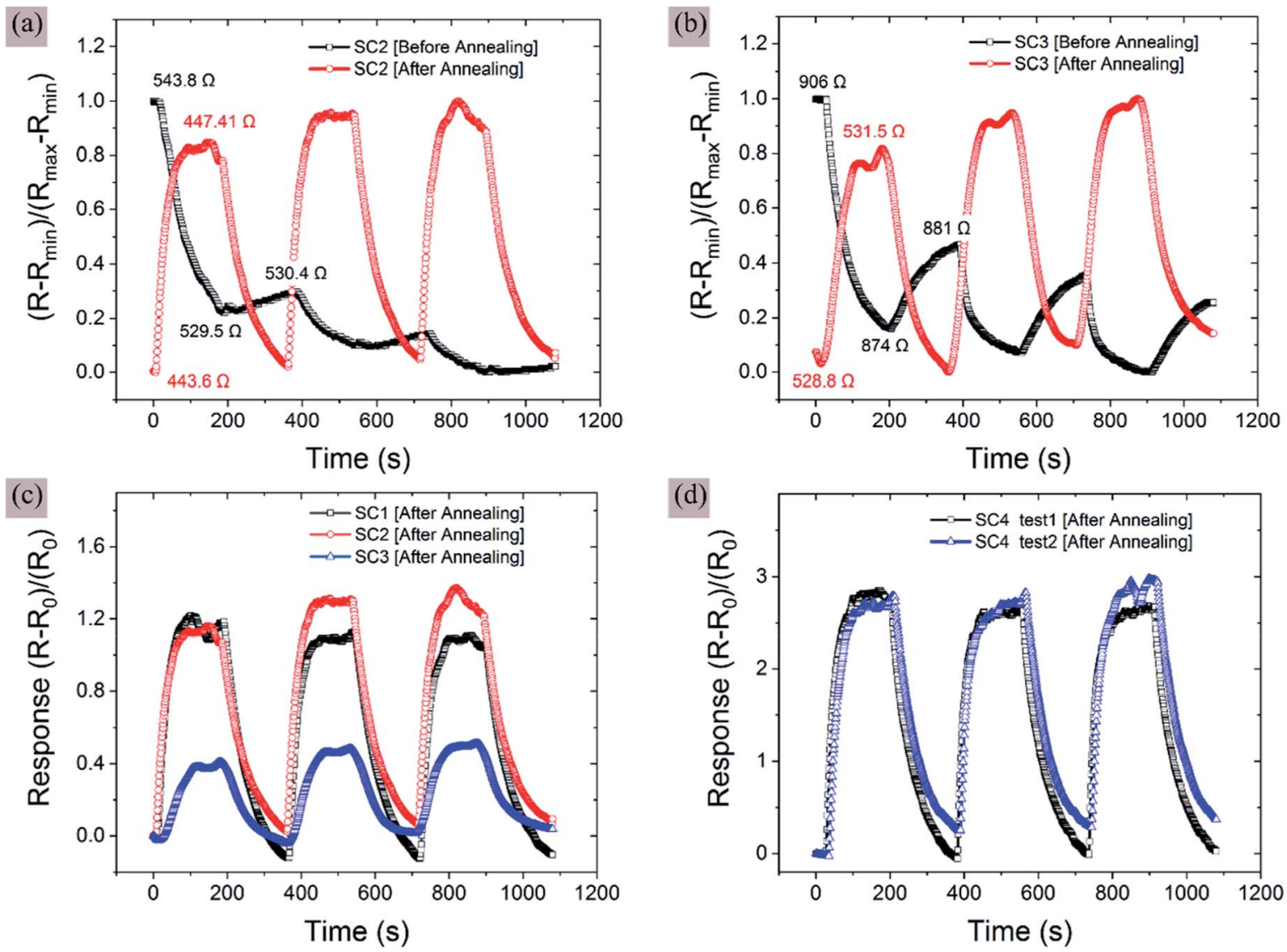

Fig. 5 The effect of thermal annealing on the sensitivity and response of the sensors. (a) Response of sensor SC2 before and after annealing. (10 min, 0.005 wt\%). (b) Response of sensor SC3 before and after annealing (10 min, 0.005 wt\%). (c) Sensitivity of sensors fabricated using MWCNTs functionalized with different functional groups (10 min, $0.005 \mathrm{wt} \%$ ). (d) Sensitivity of sensors fabricated using a mixture of MWCNTs and CAC (10 min, $0.005 \mathrm{wt} \%)$. 

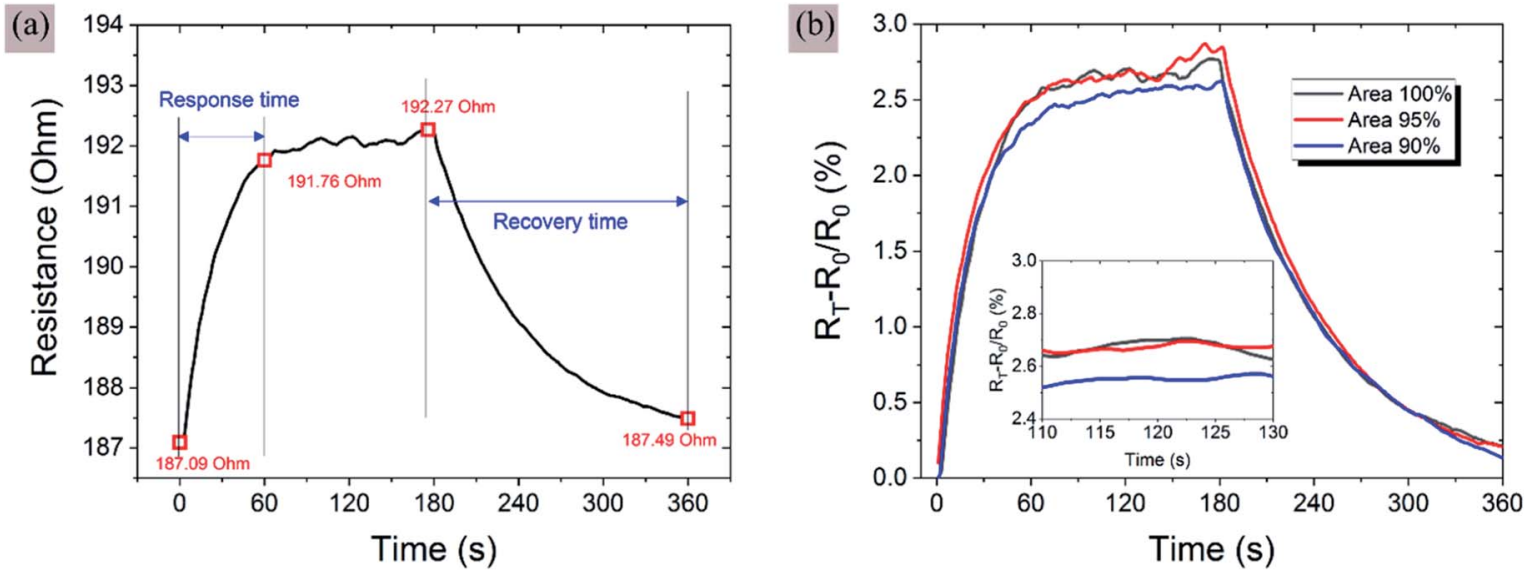

Fig. 6 Characterization of sensor SC4 (fabrication parameters: $20 \mathrm{~V}, 10 \mathrm{~min}, 0.005 \mathrm{wt} \%$ ). (a) Response and recovery time. (b) The response cycle of the sensor with reduced sensing areas.

resistance values in each cycle (Fig. 5b). However, the minimum and maximum resistance values in the first cycle were $528.8 \Omega$ and $531.5 \Omega$, respectively.

Fig. $5 \mathrm{c}$ presents the sensitivity after annealing the temperature sensors. The sensor that was fabricated using $\mathrm{COOH}-$ MWCNT (SC2) showed better sensitivity than the other sensors, while the sensor that was fabricated with $\mathrm{COOH}-\mathrm{OH}-\mathrm{MWCNT}$ (SC3) showed the worst sensitivity. The results were consistent with the TCR obtained before the thermal annealing. The inverse relationship (positive TCR) may be due to the changes in the MWCNT metallic/semiconducting ratio within the sensing material after the annealing process. ${ }^{19}$ In addition, the annealing can reduce the oxygen content of the functionalized MWCNTs, and it is known that raw MWCNTs generally show a positive TCR, while functionalized MWCNTs show a negative TCR. The remains of the solvent inside and between the tubes before the thermal annealing might affect the temperature measurements. Fig. 5d shows that the sensitivity of the temperature sensors was improved by assembling CAC particles along with the MWCNTs. The conductive carbon particles work as junctions that link the tubes to each other and form full bridges across the gap. The sensors also showed strong repeatability, where the response cycles of the two sensors were almost identical.

The response and recovery times of the temperature sensors were also investigated. Fig. 6a presents the sensing cycle of sensor SC4 exposed to hot airflow with a temperature of $\sim 60^{\circ} \mathrm{C}$. The sensor resistance reached $90 \%$ of its maximum resistance in 60 seconds. However, the sensor required more than 3 minutes to recover to $7.7 \%$ of its initial resistance.

The resistance mismatch was investigated at different sensing areas of sensor SC4 to confirm that the AA-DEP method assembled and aligned the MWCNTs across the ITO electrodes uniformly. The total gap area of the fabricated IDE electrodes was $6 \mathrm{~mm} \times 50 \mu \mathrm{m}=300000 \mu \mathrm{m}^{2}$. The initial resistance of the MWCNT bridges that coated this area was $187.09 \Omega$. The area was then reduced to $285000 \mu \mathrm{m}^{2}$ by wiping $5 \%$ of the aligned bridges. The new initial resistance of the sensor increased to $199.25 \Omega$. However, the sensor's sensitivity was identical and did not change when the sensing area was reduced. The sensing area was further reduced by $5 \%$ to result in a sensing area of $270000 \mu \mathrm{m}^{2}$. The new initial resistance was $209.56 \Omega$, and the sensor response was also similar to the response of the total area (Fig. 6b). We conclude that the AADEP method showed good reproducibility where the sensor's performance was identical although after reducing the sensing area.

\section{Conclusion}

In this work, the dielectrophoretic assembly of MWCNTs was assisted with airflow to improve the alignment quality and reduce the mismatch in the characteristics of temperature sensors. The airflow was used to improve the DEP controllability by speeding up the drying process while maintaining good alignment quality. The proposed low-cost method successfully aligned long MWCNT bridges across transparent electrodes. The best alignment results were obtained at airflow velocities between $1.5 \mathrm{~m} \mathrm{~s}^{-1}$ and $2.5 \mathrm{~m} \mathrm{~s}^{-1}$. The variation in the resistance of the sensors was tested at different fabrication parameters. The minimum variation in the resistance of the aligned bridges was $1.81 \%$ observed at MWCNT concentration of $0.005 \mathrm{wt} \%$ and deposition time of $10 \mathrm{~min}$. The long MWCNT bridges have low resistance and large contact points, making them suitable to operate as temperature sensors. The behavior of the temperature sensors after annealing was entirely different in contrast to their behavior before annealing. The characteristics of the sensors were investigated using MWCNTs functionalized with different functional groups at different concentrations. The ability of the developed method to reduce the resistance mismatch has potential use in the fabrication of CNT-based sensors.

\section{Conflicts of interest}

There are no conflicts to declare. 


\section{Acknowledgements}

This work was supported by the Universiti Putra Malaysia under Grant GPB/2017/9563800. Also, the authors would like to express their heartfelt gratitude to Hadhramout Foundation for supporting this research.

\section{References}

1 Q. Huang, Y. Jiang, Z. Duan, Z. Yuan, B. Liu, Q. Zhao, Y. Zhang, Y. Sun, P. Sun and H. Tai, J. Phys. D: Appl. Phys., 2021, 54, 284003.

2 A. Abdulhameed, I. A. Halin, M. N. Mohtar, M. N. Mohtar and M. N. Hamidon, IEEE Sens. J., 2021, 21(23), 26594-26601.

3 Z. Duan, Y. Jiang, Q. Huang, S. Wang, Y. Wang, H. Pan, Q. Zhao, G. Xie, X. Du and H. Tai, Smart Mater. Struct., 2021, 30, 055012 .

4 A. Abdulhameed, M. N. Mohtar, M. N. Hamidon and I. A. Halin, Electrophoresis, 2021, DOI: 10.1002/ elps.202100268.

5 V. Schroeder, S. Savagatrup, M. He, S. Lin and T. M. Swager, Chem. Rev., 2019, 119, 599-663.

6 X. Gong, L. Zhang, Y. Huang, S. Wang, G. Pan and L. Li, RSC Adv., 2020, 10, 22222-22229.

7 C. Li and G. Shi, J. Photochem. Photobiol., C, 2014, 19, 20-34. 8 N. Sinha, J. Ma and J. T. W. Yeow, J. Nanosci. Nanotechnol., 2006, 6, 573-590.

9 K. Xu, X. Tian, C. Wu, J. Liu, M. Li, Y. Sun and F. Wei, Sci. China: Technol. Sci., 2013, 56, 32-35.

10 X. Li, M. Le Thai, R. K. Dutta, S. Qiao, G. T. Chandran and R. M. Penner, ACS Sens., 2017, 2, 282-289.

$11 \mathrm{~J}$. Suehiro, N. Ikeda, A. Ohtsubo and K. Imasaka, IET Nanobiotechnol., 2009, 3, 15-22.

12 W. Shao, M. R. Shurin, S. E. Wheeler, X. He and A. Star, ACS Appl. Mater. Interfaces, 2021, 13, 10321-10327.

13 Y. Li, T. Wu and M. Yang, Sens. Actuators, B, 2014, 203, 63-70.

14 B. F. Monea, E. I. Ionete, S. I. Spiridon, A. Leca, A. Stanciu, E. Petre and A. Vaseashta, Sensors, 2017, 17, 2071.

15 G. Xiao, H. Weng, L. Ge and Q. Huang, Front. Mater., 2020, 7, 341.

16 B. F. Monea, E. I. Ionete, S. I. Spiridon, D. Ion-Ebrasu and E. Petre, Sensors, 2019, 19, 2464.

17 G. Matzeu, A. Pucci, S. Savi, M. Romanelli and F. Di Francesco, Sens. Actuators, A, 2012, 178, 94-99.

18 A. Di Bartolomeo, M. Sarno, F. Giubileo, C. Altavilla, L. Iemmo, S. Piano, F. Bobba, M. Longobardi, A. Scarfato, D. Sannino, A. M. Cucolo and P. Ciambelli, J. Appl. Phys., 2009, 105, 064518.

19 C. Y. Kuo, C. L. Chan, C. Gau, C. W. Liu, S. H. Shiau and J. H. Ting, IEEE Trans. Nanotechnol., 2007, 6, 63-69.

20 E. I. Ionete, A. E. Niculescu, S. I. Spiridon and B. F. Monea, IEEE Sens. J., 2021, 21, 2767-2774.

21 M.-C. Hsu and G.-B. Lee, IET Nanobiotechnol., 2014, 8, 44-50.
22 S. Gong, Z. H. Zhu and Z. Li, Phys. Chem. Chem. Phys., 2017, 19, 5113-5120.

23 M. Sibinski, M. Jakubowska and M. Sloma, Sensors, 2010, 10, 7934-7946.

24 B. Zhao, Y. Wang, S. Sinha, C. Chen, D. Liu, A. Dasgupta, L. Hu and S. Das, Nanoscale, 2019, 11, 23402-23415.

25 M. T. S. Chani, A. M. Asiri, K. S. Karimov, M. Bashir, S. B. Khan and M. M. Rahman, Int. J. Electrochem. Sci., 2014, 10, 3784-3791.

26 Y. Kumaresan, O. Ozioko and R. Dahiya, IEEE Sens. J., 2021, 21(23), 26243-26251.

27 A. Abdulhameed, N. Z. A. Wahab, M. N. Mohtar, M. N. Hamidon, S. Shafie and I. A. Halin, J. Electron. Mater., 2021, 50, 3207-3221.

28 M. S. Sadi, M. Yang, L. Luo, D. Cheng, G. Cai and X. Wang, Cellulose, 2019, 26, 6179-6188.

29 B. Zhao, V. S. Sivasankar, A. Dasgupta and S. Das, ACS Appl. Mater. Interfaces, 2021, 13, 10257-10270.

30 V. S. Turkani, D. Maddipatla, B. B. Narakathu, B. J. Bazuin and M. Z. Atashbar, Sens. Actuators, A, 2018, 279, 1-9.

31 M. T. S. Chani, K. S. Karimov and A. M. Asiri, J. Mater. Sci.: Mater. Electron., 2019, 30, 6419-6429.

32 Y. Jang, S. M. Kim, G. M. Spinks and S. J. Kim, Adv. Mater., 2020, 32, 1902670.

33 Z. Pan, Y. Zhang, Z. Cheng, J. Tong, Q. Chen, J. Zhang, J. Zhang, X. Li and Y. Li, Sensors, 2017, 17, 473.

34 W. Gao and J. Kono, R. Soc. Open Sci., 2019, 6, 181605.

35 T. Han, A. Nag, S. Chandra Mukhopadhyay and Y. Xu, Sens. Actuators, A, 2019, 291, 107-143.

$36 \mathrm{H}$. Pathangi, G. Groeseneken and A. Witvrouw, Microelectron. Eng., 2012, 98, 218-221.

37 C. K. M. Fung, V. T. S. Wong, R. H. M. Chan and W. J. Li, IEEE Trans. Nanotechnol., 2004, 3, 395-403.

38 T. Zhou, E. Kropp, J. Chen and L. Kulinsky, Micromachines, 2020, 11, 371.

39 A. Abdulhameed, M. N. Mohtar, M. N. Hamidon and I. A. Halin, Fullerenes, Nanotubes, Carbon Nanostruct.s, 2021, 29, 832-839.

40 A. Abdulhameed, M. Nazim Mohtar, M. N. Hamidon, I. Mansor and I. A. Halin, Mater. Res. Express, 2021, 8, 55603.

41 Z. Duan, Y. Jiang, S. Wang, Z. Yuan, Q. Zhao, G. Xie, X. Du and H. Tai, ACS Sustainable Chem. Eng., 2019, 7, 1747417481.

42 E. M. Remillard, Z. Branson, J. Rahill, Q. Zhang, T. Dasgupta and C. D. Vecitis, Nanoscale, 2017, 9, 6854-6865.

43 A. Abdulhameed, M. N. Mohtar, M. N. Hamidon, I. Mansor and I. A. Halin, Microelectron. Eng., 2021, 247, 111597.

44 Y. Huang, X. Gong, Y. Meng, Z. Wang, X. Chen, J. Li, D. Ji, Z. Wei, L. Li and W. Hu, Nat. Commun., 2021, 12, 1-9.

45 N. H. A. Aziz, H. Jaafar, R. M. Sidek, S. Shafie, M. N. Hamidon and R. Wagiran, J. Nanoelectron. Optoelectron., 2021, 16, 569576.

46 J. Tersoff, Appl. Phys. Lett., 1999, 74, 2122-2124. 